\title{
EFECTO DE LA TEMPERATURA DEL SEMEN SOBRE LA RESPUESTA REPRODUCTIVA DE CERDAS
}

\author{
EFFECT OF SEMEN TEMPERATURE ON THE REPRODUCTIVE \\ ANSWER OF SOWS
}

\author{
D. Alemán; M. Alfaro ${ }^{1}$; E. Hurtado
}

\begin{abstract}
RESUMEN
Con el fin de evaluar el efecto de la temperatura del semen en cerdas nulíparas, primíparas y multíparas, sobre la respuesta reproductiva, se utilizaron 91 cerdas mestizas de las razas Yorkshire y Landrace, en una granja comercial ubicada en Sabaneta, Estado Monagas, Venezuela. Se utilizó un diseño completamente al azar con arreglo factorial de tratamientos $3 \times 2$. Los factores identificados fueron: categoría de parto (nulíparas, primíparas y multíparas) y temperatura del semen $\left(37^{\circ} \mathrm{C}\right.$ y $\left.16-18{ }^{\circ} \mathrm{C}\right)$. Las variables dependientes medidas fueron: Porcentaje de partos (PP), número de lechones nacidos vivos (NLNV) y número de lechones nacidos totales (NLNT). Los da tos se estudiaron mediante análisis de la varianza, por el método de los mínimos cuadrados, con un modelo lineal de efectos fijos, para desigual número de observaciones. Se observaron el efecto $(\mathrm{P}<0,05)$ de la temperatura del semen y la interacción categoría de parto por temperatura del semen sobre el porcentaje de partos. Los valores promedios encontrados fueron: $79,13 \%$ para $37^{\circ} \mathrm{C}$ y $55,42 \%$ para $16-18{ }^{\circ} \mathrm{C}$; mientras que el mayor promedio $(85,57 \%)$ en la interacción ocurrió en las cerdas nulíparas inseminadas con semen a $37^{\circ} \mathrm{C}$. El NLNV y NLNT no presentaron diferencias $(\mathrm{P}>0,05)$ entre los factores bajo estudio. En conclusión, la interacción categoría de parto por temperatura del semen afecta la respuesta reproductiva (porcentaje de partos).
\end{abstract}

Palabras clave: Cerdas, IA, temperatura del semen, respuesta reproductiva.

\section{ABSTRACT}

With the purpose of evaluating the effect of semen temperatures on the reproductive answer of nuliparous, primiparous and multiparous sows, there were used 91 Yorkshire and Landrace half-breed sows, in a commercial farm located in Sabaneta, Monagas state, Venezuela. The treatments were distributed in a factorial arrangement (3x2) on a complete random design. The factors identified were the following: categories of parturition (nuliparous, primiparous and multiparous) and semen temperatures $\left(37^{\circ} \mathrm{C}\right.$ and $\left.16-18^{\circ} \mathrm{C}\right)$. The dependent variables were farrowing rate $(F R)$, number of piglets born alive (NPBA) and number of total born piglets $(N T B P)$. The data were analyzed through variance analysis by the method of the minimum squares, with a lineal model of fixed effects, for an unequal number of observations. It was observed an effect $(P<0.05)$ semen temperature and interaction categories of parturition $x$ semen temperature on the farrowing rate. The average values found were $79.13 \%$ for $37^{\circ} \mathrm{C}$ and $55.42 \%$ for $16-18{ }^{\circ} \mathrm{C}$, while the largest average (85.57\%) in interactions occurred in nuliparous sows inseminations with semen at $37^{\circ} \mathrm{C}$. The NPBA and NTBP did not present difference $(P>0.05)$ in factors of study. In conclusion, interaction categories of parturition $x$ semen temperature affect reproductive answer (farrowing rate).

Key words: Sows, IA, semen temperature, reproductive answer.

\section{INTRODUCCIÓN}

El éxito de un sistema de explotación porcina depende principalmente de un manejo reproducti- vo adecuado. En el ámbito mundial y nacional la inseminación artificial porcina es una técnica que ha innovado los sistemas de producción de carne de cerdo.

1 Universidad de Oriente. Escuela de Zootecnia. Departamento Producción Animal.

2 Universidad de Oriente. Escuela de Zootecnia Departamento de Biología y Sanidad Animal. Apartado Postal 6201 Maturín, Monagas, Venezuela.

e-mail: malfaro@monagas.udo.edu.ve ernestohurtado@cantv.net

Fecha de Recepción: 03 Abril 2006

Fecha de Aceptación: 10 Mayo 2006 
La evolución sostenida de esta técnica en la práctica se ha debido a los resultados obtenidos a nivel de granjas y la disminución de los costos de producción. Esta ha experimentado nuevos avances, que van desde sus inicios basados en el uso de semen a temperatura de $37{ }^{\circ} \mathrm{C}$ hasta la utilización de sistemas de inseminación artificial intrauterina con bajas dosis seminales. Por otro lado, tales avances tecnológicos contribuyen a establecer una ganadería porcina rentable, como consecuencia de una eficiente producción anual de carne, la cual se obtiene como resultado de un mayor número de lechones producidos por cerda al año.

En Venezuela, desde los años 70 la inseminación artificial a nivel experimental ha tenido resultados satisfactorios, lo que dio base a la implementación de la técnica a nivel de granjas comerciales (Fuentes, 2000).

Actualmente en el mundo el $99 \%$ de las inseminaciones realizadas emplea un método de conservación en donde el semen permanece de uno a cinco días a temperatura de 15 a $20^{\circ} \mathrm{C}$, así, el semen se conserva de forma ideal. Por debajo de $14{ }^{\circ} \mathrm{C}$ se presentan alteraciones de la membrana del espermatozoide repercutiendo en el poder fecundante del mismo. Temperaturas por encima de los $20^{\circ} \mathrm{C}$ disminuyen enormemente la vida útil del semen (Decuadro, 2001). Lo anterior indica la importancia que la temperatura tiene en la preservación de la calidad del semen. Variaciones de 1 a $2{ }^{\circ} \mathrm{C}$ pueden afectar la calidad del semen, ya que el semen porcino es particularmente sensible a los cambios térmicos, por lo que es vital conservarlo a $17^{\circ} \mathrm{C}$, y evitar fluctuaciones en la temperatura (PIC, 2001).

La conservación del semen refrigerado depende básicamente del diluyente, ya que contribuye a preservar las características funcionales de las células espermáticas manteniendo el nivel de fertilidad del eyaculado. Fuentes (2001) hace referencia sobre la conservación a temperatura de $15^{\circ} \mathrm{C}$ del semen, refrigerado con diluyente que tiene sustancias crioprotectoras (yema de huevo y leche). Además, el uso de BTS (diluyente ampliamente conocido en Europa y actualmente en Venezuela), lo cual permite mantener viable el material espermático a $17^{\circ} \mathrm{C}$ durante unos 5 días, con un porcentaje de preñez en muchos casos superior al $80 \%$.

La literatura reporta una serie de resultados que indican la importancia de la temperatura del semen en la respuesta reproductiva de la cerda. Tonieto et al. (2003) señalaron como práctica común el uso de semen conservado a una temperatura entre 15$18{ }^{\circ} \mathrm{C}$ hasta el tercer día después de la colecta y la dilución, debido a las dificultades de mantenimiento y almacenamiento de semen por un lapso mayor a tres días, por lo cual no suelen usarse con frecuencia ni el semen conservado por más de tres días ni el semen congelado. Sánchez (2003) determinó que la temperatura adecuada de conservación de semen es de $15^{\circ} \mathrm{C}-20{ }^{\circ} \mathrm{C}$, a pesar de que el porcentaje de parto pudiera verse comprometido al disminuir el metabolismo de la célula espermática. Mientras que Wayne (2002) recomienda inseminar las cerdas con semen fresco conservado durante $24-48 \mathrm{~h}$ en nevera a una temperatura entre $17-18{ }^{\circ} \mathrm{C}$ para alcanzar las más altas tasas de concepción y buen tamaño de la camada.

Todo indica que mediante el uso de la inseminación artificial, los productores y estudiosos del cerdo tienen la posibilidad de obtener grandes provechos, permitiendo adquirir de esta forma destreza y práctica en el manejo de una técnica que día a día es utilizada a nivel mundial. En este sentido, el objetivo de este trabajo fue estudiar la respuesta reproductiva en cerdas de distintos partos utilizando dos temperaturas de inseminación.

\section{MATERIALES Y MÉTODOS}

\section{UNIDAD DE PRODUCCIÓN Y ANIMALES}

En una granja comercial de ciclo completo, ubicada en Sabaneta, Estado Monagas, Venezuela, localizada a $09^{\circ} 45^{\prime}$ de Latitud Norte y $63^{\circ} 27^{\prime}$ de Longitud Oeste, y a una altura de 145 metros sobre el nivel del mar; se evaluaron 91 cerdas mestizas de las razas Yorkshire y Landrace de diferente número de partos, las cuales recibieron dos servicios. Se utilizó semen proveniente de verracos terminadores de las razas Duroc, Yorkshire, Landrace y Hampshire pertenecientes a la granja.

\section{COLECTA DE SEMEN \\ Y MANEJO REPRODUCTIVO}

El semen se recolectó durante dos períodos del día (6:30 a.m. y 5:00 p.m.), de acuerdo a la técnica empleada en la granja. Posteriormente se procedió a la evaluación seminal: macroscópica ( $\mathrm{pH}$, color y volumen) y microscópica (vitalidad, motilidad, atipias espermáticas y concentración). 
Se realizó la dilución del semen en 46,45 g de diluyente comercial, Beltsville Thawing Solution (BTS), el cual fue previamente diluido en un litro de agua destilada y calentado en un matraz a baño maría. Una vez diluido el semen, se procedió a envasar con dosis de $70 \mathrm{~mL}$. Para efecto de la IA tradicional se usó inmediatamente. En el caso del semen conservado, las dosis, una vez envasadas, se dejaron reposar por 30 minutos y luego fueron llevadas a una nevera equipada con un termostato que permitió mantener la temperatura entre 16 a $18{ }^{\circ} \mathrm{C}$. Para ambos tratamientos se utilizaron $5 \times 10^{9}$ espermatozoides por dosis.

Se realizaron dos detecciones de celo (a.m. y p.m.). La inseminación con semen a $37{ }^{\circ} \mathrm{C}$ (tradicional) y $16-18{ }^{\circ} \mathrm{C}$ fue efectuada a las 12 y 24 horas después de confirmado el celo.

\section{ANÁLISIS ESTADÍSTICO}

El diseño utilizado fue completamente al azar con un arreglo de tratamientos factorial $3 \mathrm{x}$ 2 siendo los factores o variables independientes: Categoría de parto (14 nulíparas, 23 primíparas y 54 multíparas) y temperatura del semen $\left(37^{\circ} \mathrm{C}\right.$ y $16-18^{\circ} \mathrm{C}$ ). Las variables dependientes a medir fueron: porcentaje de partos, número de lechones nacidos vivos y número de lechones nacidos totales. Los datos obtenidos se analizaron a través de ANAVA por medio del procedimiento GLM (SAS, 1998), para desigual número de observaciones, y se realizaron comparaciones múltiples (mínima diferencia significativa) a los factores representativos. Para evaluar el porcentaje de partos se le asignó el valor de uno (1) a la cerda que parió y cero (0) a la cerda que no parió y se reportó como porcentaje: (Número de cerdas que parieron / número de cerdas servidas) por 100.
El modelo lineal aditivo con efectos fijos fue:

$$
\mathrm{Y}_{\mathrm{ijk}}=\mu+\alpha_{\mathrm{i}}+\beta_{\mathrm{j}}+(\alpha \beta)_{\mathrm{ij}}+\varepsilon_{\mathrm{ijk}}
$$

Donde:

$\mathrm{Y}_{\mathrm{ijk}}=$ Observación k-ésima del i-ésimo nivel del factor A (temperatura del semen) y j-ésimo nivel del factor B (categoría de parto).

$\mu \quad=$ media teórica de la población.

$\alpha_{\mathrm{i}}=$ Efecto de la temperatura del semen "i", donde "i" $=(1-2)$.

$\beta_{\mathrm{j}} \quad=$ Efecto del categoría de parto " $\mathrm{j}$ ", donde "j" = (1...3).

$(\alpha \beta)_{\mathrm{ij}}=$ Efecto de interacción temperatura del semen "i" con la categoría de parto " $\mathrm{j}$ ".

$\varepsilon_{\mathrm{ijk}}=$ Efecto del error experimental con media cero y varianza común.

\section{RESULTADOS Y DISCUSIÓN}

Las medias ajustadas y los errores estándares de las variables: porcentaje de partos, número de lechones nacidos vivos y número de lechones nacidos totales se muestran en la Tabla 1. Estos resultados generales en el porcentaje de partos difieren con los reportados por Millán (1996), Alfaro (1996) y Zapata (1996) posiblemente debido a la aplicación de la técnica de la inseminación artificial, mientras que las otras variables concuerdan con lo reportado por la literatura.

\section{PORCENTAJE DE PARTOS}

El porcentaje promedio de partos calculado fue afectado significativamente $(\mathrm{P}<0,05)$ por la temperatura del semen y la interacción categoría de parto $\mathrm{x}$ temperatura del semen.

Tabla 1

Medias ajustadas y errores estándares para las variables estudiadas

\begin{tabular}{lccc}
\hline Variable & Número de cerdas & Media & Error estándar \\
\hline Porcentaje de partos & 91 & 67,03 & 4,49 \\
Número de lechones nacidos vivos & 61 & 8,68 & 2,62 \\
Número de lechones nacidos totales & 61 & 9,57 & 2,56 \\
\hline
\end{tabular}


La temperatura del semen utilizada de $37^{\circ} \mathrm{C}$ arrojó el mayor promedio de porcentaje de partos (Tabla 2). Sin embargo, resultados superiores fueron reportados por Alfaro (1996) en cerdas inseminadas con semen a $37^{\circ} \mathrm{C}$, mientras que experiencias recientes realizadas por Leyún (2004) han demostrado la efectividad del uso de semen a $17^{\circ} \mathrm{C}$ al compararlo con el semen aplicado a $37^{\circ} \mathrm{C}$. Es importante señalar que esta diversidad de resultados está asociada a la viabilidad espermática como consecuencia posiblemente de los efectos ambientales.

El comportamiento de la interacción categoría de parto por temperatura del semen se presenta en la Tabla 3. Se observa que los mayores valores lo presentaron las cerdas nulíparas inseminadas con semen a $37^{\circ} \mathrm{C}$ y las cerdas multíparas inseminadas con semen entre 16 y $18{ }^{\circ} \mathrm{C}$. Sin embargo, esta leve superioridad de las cerdas nulíparas a $37^{\circ} \mathrm{C}$ pudiera atribuirse al bajo número de observaciones, debido a que las cerdas mantuvieron su comportamiento independiente de la temperatura del semen. La mejor respuesta en las cerdas multíparas sobre las primíparas coincide con lo reportado por Leyún (2004).

Número de lechones nacidos vivos y número de lechones nacidos totales

Los efectos temperatura del semen, categoría de parto y la interacción entre ellos, resultaron no significativos para las variable número de lechones nacidos vivos y número de lechones totales $(\mathrm{P}>0,05)$. Sin embargo, los mayores valores encontrados para número de lechones nacidos vivos $(8,83)$ y número de lechones nacidos totales $(10,01)$ ocurrieron en las cerdas multíparas que fueron inseminadas con semen a temperatura $37^{\circ} \mathrm{C}$.

Estos resultados son similares a los reportados por Alfaro (1996) y Millán (1996). Mientras que Leyún (2004) reportó para el tamaño de la camada en cerdas multíparas 12,26 (con semen a $37^{\circ} \mathrm{C}$ ) y 11,70 (con semen a $17^{\circ} \mathrm{C}$ ), no encontrando diferencias estadísticas para la temperatura de inseminación con respecto al tamaño de la camada.

Tabla 2

Efecto de la temperatura del semen sobre el porcentaje de partos

\begin{tabular}{cccc}
\hline Temperatura del semen & n & Media & Error estándar \\
\hline $37^{\circ} \mathrm{C}$ & 47 & $79,13^{\mathrm{a}}$ & 0,73 \\
$16-18^{\circ} \mathrm{C}$ & 44 & $55,42^{\mathrm{b}}$ & 0,78 \\
\hline
\end{tabular}

Medias con letras diferentes son estadísticamente significativas $(\mathrm{P}<0,05)$.

Tabla 3

Medias mínimos cuadráticas y error estándar de las combinaciones temperatura del semen por categoría de parto para el porcentaje de partos

\begin{tabular}{ccccc}
\hline Temperatura del semen & Categoría de parto & $\mathbf{n}$ & $\begin{array}{c}\text { Porcentaje promedio } \\
\text { de partos }\end{array}$ & Error estándar \\
\hline $37^{\circ} \mathrm{C}$ & Nulíparas & 7 & $85,57^{\mathrm{a}}$ & 1,64 \\
$37^{\circ} \mathrm{C}$ & Primíparas & 14 & $64,57^{\mathrm{a}}$ & 1,16 \\
$37^{\circ} \mathrm{C}$ & Multíparas & 26 & $69,07^{\mathrm{a}}$ & 0,85 \\
$16-18^{\circ} \mathrm{C}$ & Nulíparas & 7 & $28,57^{\mathrm{b}}$ & 1,64 \\
$16-18^{\circ} \mathrm{C}$ & Primíparas & 9 & $44,57^{\mathrm{ab}}$ & 1,45 \\
$16-18^{\circ} \mathrm{C}$ & Multíparas & 28 & $78,14^{\mathrm{a}}$ & 0,82 \\
\hline
\end{tabular}

Medias con letras diferentes son estadísticamente significativas $(\mathrm{P}<0,05)$. 


\section{CONCLUSIONES}

- Los resultados obtenidos permiten concluir la evidencia de una relación de dependencia entre la temperatura del semen y categoría de parto para la variable porcentaje de partos. No así para número de lechones nacidos vivos y nacidos totales. Se recomienda realizar nuevas investigaciones a fin aportar resultados que se puedan comparar con esta experiencia.

\section{LITERATURA CITADA}

ALFARO, M. (1996). Parámetros productivos y tasa de parición de cerdas primíparas y multíparas sometidas a monta natural e inseminación artificial. Trabajo de Ascenso. Escuela de Zootecnia. Universidad de Oriente. Maturín, Venezuela. 90 p.

DECUADRO, G. (2001). Avances en inseminación artificial porcina. [Documento en línea]. Disponible en: http://www. acontece.com.ar Consulta:25/04/2001.

FUENTES, A. (2000). Inseminación Artificial Porcina en Venezuela. Instituto de Investigaciones Zootécnicas. Fondo Nacional de Investigaciones Agropecuarias. Maracay, Venezuela. Serie C. No $47.71 \mathrm{p}$.

FUENTES, A. (2001). Resultados experimentales en el manejo reproductivo del verraco. [Documento en línea]. Disponible en:http://www.ceniap.gov.ve/bdigital/monografias/verraco/ verracomonografia.htm Consulta: 10/01/2003.

LEYÚN, M. (2004). Estudio comparado de diferentes sistemas de aplicación de semen porcino. Revista Navarra Agraria. ITG. Ganadero. 144: 41-48.

MILLÁN, J. (1996). Estudio de parámetros productivos y reproductivos en cerdas primíparas y multíparas sometidas a monta natural e inseminación artificial. Trabajo de Grado. Escuela de Zootecnia. Universidad de Oriente. Maturín, Venezuela. $106 \mathrm{p}$.

PIC. PIG IMPROVEMENT COMPANY (2001). Conservación de la calidad del semen: Diluyente, empaque, temperatura y transporte. [Documento en línea].Disponible en: www. porcicultura.com Consulta: 03/11/2001.

SÁNCHEZ, R. (2003). Conceito e fundamento dos diluyentes utilizados na inseminação artificial en suinos. Suinos \& Cia Revista Especializada em Reprodução Suina. 2: 18-22.

SAS. STATISTICAL ANALYSIS SYSTEM (1998). User's Guide Statistics. (Version 6.01). SAS. Int. Inc. Cary, N.C.

TONIETO, S.; C. GONÇALVES; M. NUNES; T. LUCIA; E. I. BIANCHI (2003). Composiçao e funçoes de diluentes para o acondicionamento de semen suino.Suinos \& Cia. Revista Técnica de Suinocultura. 5: 14-16.

WAYNE, S. (2002). Guía básica para la recolección del semen porcino. Evaluación y Procesamiento. [Documento en línea]. Disponible en: http://www.porcicultura.com Consulta: 18/01/2001.

ZAPATA, M. (1996). Evaluación de parámetros productivos y reproductivos en cerdas primíparas y multíparas servidas con monta natural e inseminación artificial. Trabajo de Grado. Escuela de Zootecnia. Universidad de Oriente. Maturín, Venezuela. $117 \mathrm{p}$. 